\title{
Analysis of Direction of Arrival Techniques Using Uniform Linear Array
}

\author{
S. N. Bhuiya, F. Islam, and M. A. Matin
}

\begin{abstract}
The performance of smart antennas relies heavily on the accurate estimation of the direction of arrival (DOA). This paper analyzed various techniques for DOA estimation and investigated their performance. Simulation results showed that MUSIC algorithm outperforms in terms of root mean square error (RMSE), angular resolution and the number of elements in an antenna array. Profound analysis of these algorithms can be used to determine the direction of arrival of the signals at uniform linear array (ULA).
\end{abstract}

Index Terms-Conventional beamforming, DOA, MUSIC, MVDR, ULA.

\section{INTRODUCTION}

Using smart antennas is one of the most promising technologies that can increase the capacity in wireless networks by effectively reducing multipath and co-channel interference. Two major issues are concerned with the smart antenna - DOA estimation and the beamforming [1]. The accurate estimation of the DOA of all signals transmitted to the adaptive array antenna contributes to the maximization of its performance with respect to recovering the signal- ofinterest (SOI) and suppressing any presence of interfering signals. The beamforming technique also ensures less interference to the system and thus increasing the performance.

The DOA algorithms can be categorized into two groups: the conventional algorithm (Conventional Beamforming method, MVDR Beamforming) and the subspace algorithm (MUSIC). The conventional (Barlett) beamformer dates back to the second world-war, and is a mere application of Fourier-based spectral analysis to spatio-temporally sampled data. However, this technique works well with only one incoming signal. To overcome this problem, in 1969, the Capon's beamforming technique (Minimum Variance Algorithm) was proposed in which the array output power contains signal contributions from the desired angle as well as the undesired angles. The introduction of subspace - based estimation techniques marked the beginning of a new era in the sensor array signal processing literature [2]. The Music (Multiple Signal Classification) algorithm proposed by Schmidt in 1986, is a very popular subspace- based method for super-resolution direction finding [3].In this paper, a detailed study of different DOA algorithms has been provided and observed that MUSIC algorithm, has the least RMSE as a function of snapshots compared to the other mentioned algorithms, according to our simulation results.

Manuscript received August 2, 2012; revised September 30, 2012.

The authors are with the Dept. of Electrical Engineering and Computer Science North South University, Dhaka, Bangladesh (e-mail: shayla_298@hotmail.com).

\section{SYSTEM DESCRIPTION}

The system consists of Uniform Linear Array (ULA) with $\mathrm{M}$ number of antenna elements that are spaced linearly with equal distance.

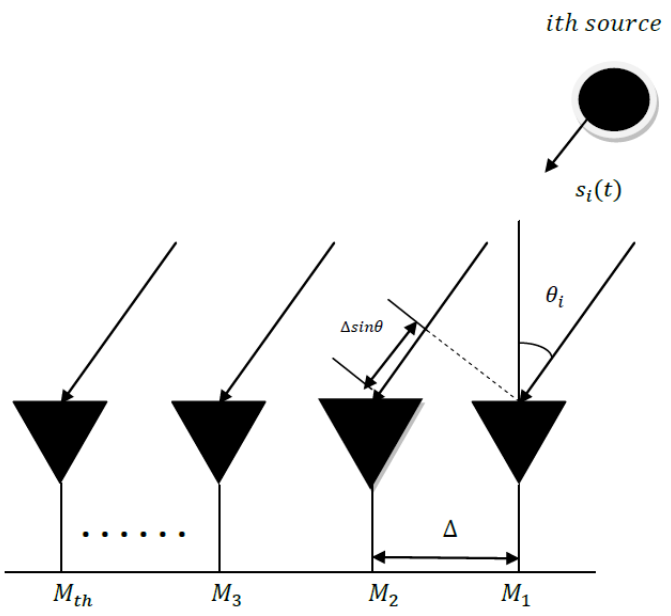

Fig. 1. System model for DOA estimation using a uniform linear array of $M$ elements.

The elements of ULA collect signals form a remote source, $i$. The spacing between the lines of elements, $\Delta$, is restricted to half wavelength of received signals. Suppose that a plane wave signal generated by the source $i$ impinges on the array at an angle $\theta_{i}$ and the signal generated by the source $i$ is the narrowband signal, $s_{i}(t)$. Then it travels at a speed of $c$ over a distance $d_{d}$ and reaches the first rightmost elements. If we consider all the signals generated by all the $d$ sources, $S_{i}(t)$, $1 \leq i \leq d$, the total signal and noises received by the $m$ th array element at time $t$ can be expressed as:

$$
x_{m}(t)=S_{i}(t) \sum_{i=1}^{d} e^{j(m-1) \mu_{i}}+n_{m}(t), m=1
$$

This equation can be written as:

$$
\begin{aligned}
x & =\left[a\left(\mu_{1}\right), a\left(\mu_{2}\right) \ldots a\left(\mu_{d}\right)\right]\left[\begin{array}{c}
S_{1}(t) \\
S_{2}(t) \\
\vdots \\
S_{d}(t)
\end{array}\right]+n(t) \\
& =A s(t)+n(t)
\end{aligned}
$$

where

$$
x(t)=\left[\begin{array}{llll}
x_{1}(t) & x_{2}(t) \ldots & x_{M}(t)
\end{array}\right]^{T}
$$

is the data column vector received by the array,

$$
s(t)=\left[\begin{array}{llll}
s_{1}(t) & s_{2}(t) \ldots & s_{M}(t)
\end{array}\right]^{T}
$$

is the signal column vector generated by the sources,

$$
n(t)=\left[\begin{array}{llll}
n(t) & n_{2}(t) & \ldots & n_{M}(t)
\end{array}\right]^{T}
$$


is a zero mean spatially uncorrelated additive noises with covariance matrix equal to $\sigma_{N}^{2} I_{M}$. The array steering column vector $a\left(\mu_{i}\right)$ is defined as:

$$
a\left(\mu_{i}\right)=\left[\begin{array}{lllll}
1 & e^{j \mu_{i}} & e^{j 2 \mu_{i}} & \ldots & e^{j(M-1) \mu_{i}}
\end{array}\right]^{T}
$$

The columns of $M \times d$ steering matrix

$$
\begin{aligned}
& A=\left[\begin{array}{lllll}
a\left(\mu_{1}\right) & \ldots & a\left(\mu_{i}\right) & \ldots & a\left(\mu_{d}\right)
\end{array}\right] \\
& =\left[\begin{array}{cccc}
1 & 1 & \ldots & 1 \\
e^{j \mu_{1}} & e^{j \mu_{2}} & \ldots & e^{j \mu_{d}} \\
\ldots & \ldots & \ldots & \ldots \\
e^{j(M-1) \mu_{1}} & e^{j(M-1) \mu_{2}} & \ldots & e^{j(M-1) \mu_{d}}
\end{array}\right]
\end{aligned}
$$

where, $\mu_{i}=-\frac{2 \pi}{\lambda} \Delta \sin \theta_{i}$, is called spatial frequency for the $i$ th source that generates the signals of incident angle, $\theta_{i}$. [3]

The idea is to 'steer' the array in one direction at a time and measure the output power. The steering direction which coincides with the DOA of a signal and result in a maximum output power yields the DOA estimates. According to [3] an array can be steered electronically just as a fixed antenna can be steered mechanically. However, the array pattern can change shape in addition to changing orientation. A weight vector $\boldsymbol{w}$ can be designed and then used to linearly combine the data received by the array elements to form a single output signal $Y(t)$,

$$
Y(t)=w^{H} X(t)
$$

The total averaged output power out of an array over $\mathrm{K}$ snapshots can be expressed as [2]

$$
\begin{gathered}
P(w)=\frac{1}{k} \sum_{k=1}^{K}\left|Y\left(t_{k}\right)\right|^{2}= \\
=\frac{1}{k} \sum_{k=1}^{K} w^{H} X\left(t_{k}\right) X^{H}\left(t_{k}\right) \\
=w^{H} R_{x x} w
\end{gathered}
$$

\section{A. Classical Beamformer}

The classical or conventional beamformer method is also referred to as the delay and sum method or Barlett method. Suppose an ULA consists of $M$ number of elements, where $\mathrm{A}(\theta)$ is defined as the steering vector with a scanning angle $\theta$. The idea is to scan across the angular region of interest, where weight vector,

$$
w=A(\theta)
$$

Inserting this equation of the weight vector into equation (9), we can calculate the output power of the classical beamformer as a function of the angle of arrival-

$$
P_{\text {Classical }}(\theta)=w^{H} R_{x x} w=A(\theta) R_{x x} A(\theta)^{H}
$$

The direction that produces the largest output power is the estimate of the desired signal's direction. [3],[4]

\section{B. Minimum Variance Distortionless Response}

MVDR is also known as the Capon's Minimum Variance method [5]. This method was proposed to overcome the poor resolution problem associated with the delay-and-sum method [6], [7]. It uses the array weights which are obtained by minimizing the mean output power with the constraint that the gain remains unity in the look direction, $\theta$ of the SOI.

The optimized weight vector is given by -

$$
w=\frac{R_{x x}^{-1} A(\theta)}{A(\theta) R_{x x}^{-1} A(\theta)^{H}}
$$

This gives the expression for the MVDR Spatial Spectrum-

$$
P_{M V}(\theta)=w^{H} R_{x x} w=\frac{1}{A(\theta) R_{x x}^{-1} A(\theta)^{H}}
$$

The angle $\theta$ in spectrum corresponding to the peak value gives the true DOA estimation.

\section{Multiple Signal Classification Algorithms}

Music is a high resolution subspace DOA algorithm, which stands for multiple signal classification. An estimate $R_{x x}$ of the covariance matrix is obtained and its eigenvectors are separated into signal and noise subspace and the DOA is estimated from one of these subspaces. It is assumed that the noise in each channel is uncorrelated. This leads to a diagonal covariance matrix.

$$
R_{x x}=A(\theta) S_{s} A(\theta)^{H}+\sigma^{2} I
$$

where, $A(\theta)=\left[a\left(\theta_{1}\right), a\left(\theta_{2}\right), a\left(\theta_{3}\right), \ldots a\left(\theta_{D}\right)\right]$ is a $M \times d$ array steering matrix. $\sigma^{2}$ is the noise variance and $\mathrm{I}$ is an identity matrix of size $M \times M$

$$
R_{x x}=A(\theta) S_{s} A(\theta)^{H}+\sigma^{2} I=Q \wedge Q^{H}
$$

with $\mathrm{Q}$ unitary and a diagonal matrix $\Lambda=\operatorname{diag}\left\{\lambda_{1}, \lambda_{2}, \ldots, \lambda_{L}\right\}$, of real eigenvalue ordered as $\lambda_{1} \geq \lambda_{2} \geq \cdots \geq \lambda_{M} \geq 0$. The vector that is orthogonal to $\mathrm{A}$ is the eigenvector of $\mathrm{R}$ having the eigenvalue $\sigma^{2}$ [8].

The number of such linearly independent vectors are $L-M$. Now, the rest of the eigenvalues are greater than $\sigma^{2}$, we can divide the eigenvalue and corresponding vector pairs into the noise and signal eigenvectors.

Now we can write it as follows-

$$
R_{x x}=S_{s} \wedge_{s} S_{s}{ }^{H}+S_{n} \Lambda_{n} S_{n}{ }^{H}
$$

where, $\Lambda_{n}=\sigma^{2} I$. All noise eigenvectors are orthogonal to $A$. Therefore the columns of $S_{S}$ should span the range space of $A$ while the columns of $S_{n}$ span its orthogonal complement. The signal and noise subspace's projection operators are defined as -

$$
\begin{aligned}
& \Pi=S_{s} S_{s}{ }^{H}=A\left(A^{H} A\right)^{-1} A^{H} \\
& \prod^{\perp}=S_{n} S_{n}{ }^{H}=1-A\left(A^{H} A\right)^{-1} A^{H}
\end{aligned}
$$

MUSIC “Spatial Spectrum” is defined as [9], [10]

$$
P_{M}(\theta)=\frac{A(\theta) A(\theta)^{H}}{A(\theta) \Pi^{\perp} A(\theta)^{H}}
$$

\section{Simulation Results}

The classical, MVDR and MUSIC techniques for DOA estimations are simulated in MATLAB and their performances are demonstrated in terms of the number of elements, root mean square error as a function of snapshots and angular resolution.

The number of elements, $M$, is an important parameter that impacts on the accuracy of the DOA estimation done by three algorithms. Here, the simulation has been carried out for two different cases: $M=10$ and $M=50$ for two differentsignals arriving at $-5 M$ and $5 M$. 


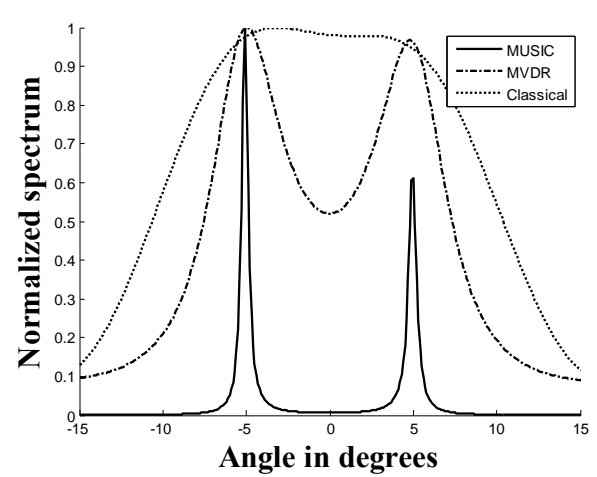

a)

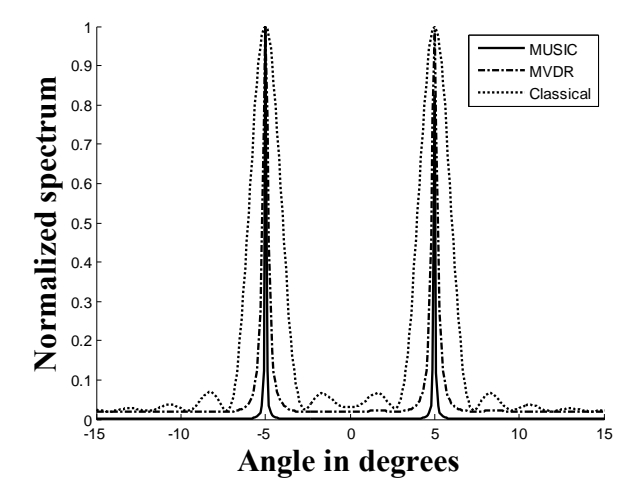

b)

Fig. 2. DOA estimation analysis for varying number of elements for: (a) $M=10$ and (b) $M=50$.

The simulated results show that increasing number of elements improves the performance of all three algorithms. However, increasing the number of elements leads to an increase in number of receivers as well as the storage capacity for the data. For less number of elements, only MUSIC algorithm gives the expected result.

Next, RMSE parameter has been used to measure the performance of the above three kinds of algorithms as a function of varying number of snapshots and it can be defined as-

$$
R M S E=\sqrt{\frac{1}{K} \sum_{k=1}^{K}\left(\theta_{\text {est }}-\theta_{\text {true }}\right)^{2}}
$$

where $\mathrm{K}$ is the total number of snapshots, $\theta_{\text {true }}$ is the true angle and $\theta_{\text {est }}$ is the angle estimated by the algorithm. For different values of $K$ we get different values of RMSE.

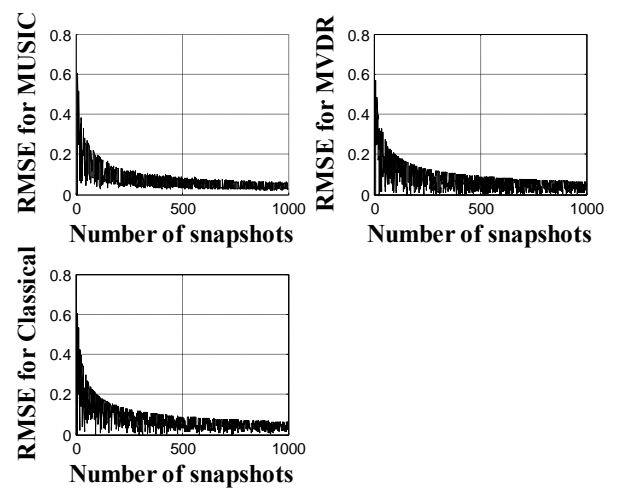

Fig. 3. RMSE for three different algorithms as a function of snapshots, for $\theta=-30 M$

The Fig depicts that as the number of snapshots increases, the resolution capability of the three algorithms increases. However, for all values of K, the MUSIC algorithm shows the least RMSE compared to the conventional algorithms-Classical and MVDR methods. This clearly indicates that the MUSIC algorithm provides the closest estimate of the true angle and therefore results in the lowest RMSE for different number of snapshots.

Finally, we analysis have done based on the resolution capability of the DOA estimation techniques considering two close signals arriving from $\theta=0 \mathrm{M}$ and $\theta=2 \mathrm{M}$ when the number of elements is 50 and the number of snapshots is 1000 .

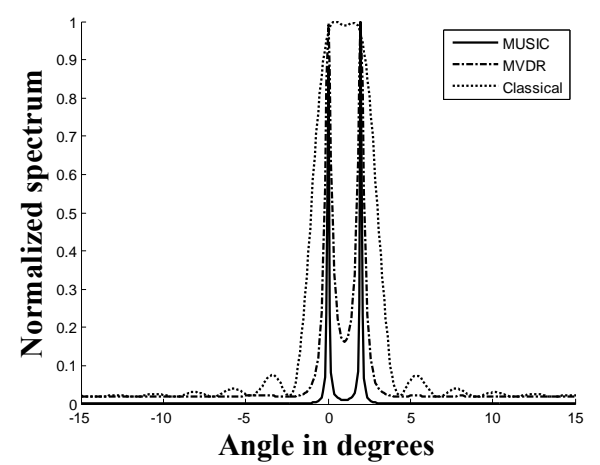

Fig. 4. DOA estimation analysis for angular resolution.

The comparison shown in Fig. 4, tells that the MUSIC algorithm has better angular resolution than MVDR which requires an additional matrix inversion. It can distinguish two received signals which are at least $2 M$ apart whereas Classical cannot.

Appendix

\begin{tabular}{|c|c|}
\hline Symbol & Meaning \\
\hline$M$ & Number of elements \\
\hline$s_{i}(t)$ & Signal from the $i$ th source \\
\hline$\theta_{i}$ & Incident angle of signal \\
\hline$d$ & Number of sources \\
\hline$x_{m}(t)$ & Total signal \& noises received by $m$ th array \\
\hline$\mu_{i}$ & Spatial Frequency \\
\hline$n(t)$ & Noise signal \\
\hline$A$ & Steering Matrix \\
\hline$A(\theta)$ & Steering vector \\
\hline$Y(t)$ & Output signal \\
\hline$w$ & Weight vector \\
\hline$w^{H}$ & Hermitian of weight vector \\
\hline$P(w)$ & Total average output power \\
\hline$K$ & Number of snapshots \\
\hline$R_{x x}$ & Covariance matrix \\
\hline$P_{\text {Classical }}(\theta)$ & Spatial spectrum of classical algorithm \\
\hline$P_{M V}(\theta)$ & Spatial spectrum of MVDR algorithm \\
\hline$P_{M}(\theta)$ & Spatial spectrum of MUSIC algorithm \\
\hline$\sigma^{2}$ & Noise variance \\
\hline$I$ & Identity matrix of size $M \times M$ \\
\hline$S_{S}$ & Signal subspace \\
\hline$S_{N}$ & Noise subspace \\
\hline
\end{tabular}

\section{CONCLUSION}

In this paper, a detail analysis of previous algorithms used to determine the direction of arrival of the signals at ULA is presented. Simulations have been performed to evaluate the performance of the classical, MVDR, MUSIC algorithms. It was observed that although increasing the number of elements improved the performance of the three algorithms, MUSIC algorithm gives the expected result with fewer 
numbers of elements. This results in an advantage of requiring less number of receivers and storage capacity at the array. These results further show that classical and MVDR algorithms offer less computational complexity. On the other hand, the MUSIC algorithm provides the most accurate DOA estimation.

\section{REFFERENCES}

[1] A. Randazzo, M. A. A. Khousa, M. Pastorino, and R. Zoughi, "Direction of arrival estimation based on Support Vector Regression: Experimental validation and comparison with MUSIC," IEEE Antennas and Wireless Propagation Letters, vol. 6, pp. 379, 2007.

[2] H. Krim and M. Viberg, "Two decades of array signal processing research," IEEE Signal Processing Magazine, vol. 13, no. 4, pp. 67, July 1996.

[3] Z. Chen, G. Gokeda, and Y. Yu, Introduction to Direction-of-Arrival Estimation, Artech House, vol. 34, no. 37, pp. 46-47, 2010.
[4] L. C. Godara, "Application of antenna arrays to mobile communications-II: Beam-forming and direction-of-arrival considerations," Proc. IEEE, vol. 85, no. 8, pp. 1195-1245, Aug. 1997.

[5] J. Capon, "High resolution frequency-wave number spectrum analysis," Proc. IEEE, vol. 57, no. 8, Aug. 1969.

[6] C. A. Balanis and P. I. Ioannides, "Introduction to smart antenna," Morgan and Claypool Publishers, 2007, pp.79.

[7] P. Stoica, Z. Wang, and J. Li, "Robust capon beamforming," IEEE Signal Processing Letters, vol. 10, no. 6, June 2003.

[8] H. Anton and C. Rorres, Elementary Linear Algebra, $8^{\text {th }}$ ed. John Wiley and Sons, 2000, pp. 338-360.

[9] R. O. Schmidt, "Multiple emitter location and signal parameter estimation," IEEE Trans, vol. AP-34, no. 3, pp. 276-280, Mar. 1986.

[10] R. Kawitkar, "Performance of different types of array structures based on multiple signal classification (MUSIC) algorithm," Fifth International Conference on MEMS, NANO, and Smart Systems, pp. 2, 2009. 